\title{
MR enterography with a new negative oral contrast solution containing maghemite nanoparticles
}

\author{
Ingrid Markova a, ${ }^{\text {, }}$, Katarina Polakova ${ }^{b}$, Pavel Tucekc, Miroslav Mashlan ${ }^{d}$, Pavel Novake, Radek Zboril ${ }^{b}$, Miroslav Herman ${ }^{f}$
}

\begin{abstract}
Aim. The aim of this study was to test an oral contrast solution with maghemite for the magnetic resonance imaging of small bowel diseases.

Patients and methods. The study sample included 3 cohorts: 17 healthy volunteers (group A), 22 patients with small bowel disease (group C). Both groups underwent MR enterography and 24 patients with small bowel disease (group B) underwent magnetic resonance cholecystopancreaticography. Various concentrations in $1000 \mathrm{ml}$ vs $500 \mathrm{ml}$ of experimental solution were tested. All cohorts completed questionnaires evaluating the solution characteristics and side-efects during and after drinking.

Results. A maghemite concentration of $800 \mathrm{mg} / 4 \mathrm{~g}$ bentonite in $1000 \mathrm{ml}$ solution was sufficient for proper intraluminal lay-out. An experimental solution of $500 \mathrm{ml}$ was sufficient for magnetic resonance cholecystopancreaticography and $1000 \mathrm{ml}$ for MR enterography. There were no statistically significant differences between groups for taste, taste characteristic or appearance of the experimental solution. Side-effects experienced during drinking were: nausea (29.4\%) and eructation (29.4\%) in group A, in group B (42\%) and diarrhoea (27.3\%) in group C. Side-effects $2 \mathrm{~h}$ after drinking occured in group A (nausea 17.6\%) and in group C (diarrhoea 47\%). The best tolerance of experimental solution was found in group $B$ with a higher median patient age than groups $A$ and $C$. The experimental solution was evaluated more favorably in the older subjects (age over 50 years).
\end{abstract}

Conclusion. The experimental oral solution with maghemite was well tolerated in all 3 groups. Our study supports its use in magnetic resonance practice.

Key words: MR enterography, maghemite, MRCP

Received: May 9, 2011; Accepted with revision: January 3, 2012; Available online: January 30, 2012 http://dx.doi.org/10.5507/bp.2012.001

${ }^{a}$ Department of Radiology, F.D.Roosevelt Faculty Hospital, nam. gen. Svobody 1, 97517 Banska Bystrica, Slovak Republic ${ }^{b}$ Regional Center of Advanced Technologies and Materials, Department of Physical Chemistry, Faculty of Science, Palacky University Olomouc, Czech Republic

'Department of Geoinformatics, Faculty of Science, 17.listopadu 1192/12, 77146, Olomouc

${ }^{d}$ Center for Nanomaterial Research, Palacky University Olomouc

eMedihope s.r.o., Mathonova 291/1, 79604 Prostejov

${ }^{f}$ Department of Radiology, Faculty of Medicine and Dentistry, Palacky University Olomouc

Corresponding author: Ingrid Markova, e-mail: ingrid.markova@gmail.com

\section{INTRODUCTION}

MRI is increasingly used for assessment of small bowel diseases. MRI has a high diagnostic accuracy and reproducibility, both with enteroclysis and the oral contrast method (enterography) for evaluation of inflammatory diseases and malabsoption syndrome (celiac disease).

The dilemma between the oral approach in MR enterography (MREg), which is more acceptable to patients and the intubation-infusion method in MR enteroclysis (MREc), which is less tolerated, in the absence of conscious sedation, has not been resolved by the radiologic community ${ }^{1-3}$. Patient's more acceptable MREg has potentional intrinsic advantages over both computed enteroclysis (CTE) and capsule enteroscopy (CE) due to the avoidance of ionizing radiation exposure, safety in pregnancy, detecting extraluminal, intraluminal and perianal disease ${ }^{4}$. MREg gives excellent soft tissue resolution and allows comparison of initial evaluation and lifelong follow-up. The proper examination of small and large bowel requires intraluminal contrast agents for lumen opacity,luminal distension and high contrast resolution between the lumen and the bowel wall. Adequate bowel distension for MREg is achieved by non osmotic agents without the intubation technique and water resorption is slowed down. Contrast agents need to meet the following requirements: minimal mucosal absorption, absence of artifacts, no significant adverse effects and low cost ${ }^{5}$. There are 3 main groups of intraluminal contrast agents based on providing signal intensity depending on the used pulse sequence. Positive contrast agents (high signal on T2 and T1-weighted images) are gadolinium chelates, manganese chloride, ferrous ammonium citrates, etc. The second group includes biphasic contrast agents (low signal on T1 and high signal on T2-weighted images) like polyethylenglycol (PEG), mannitol/sorbitol, water, methylcellulose (Vidogum), (locust bean gum) (ref. $\left.{ }^{5}\right),($ VoLumen) $\left(\right.$ ref. $\left.^{6}\right)$ (Fig. 1,2). Negative contrast agents (low signal on $\mathrm{T} 2 / \mathrm{T} 2 *$ and $\mathrm{T} 1$-weighted images) are solutions with Super Paramagnetic Iron Oxides (SPIO) nanoparticles for 


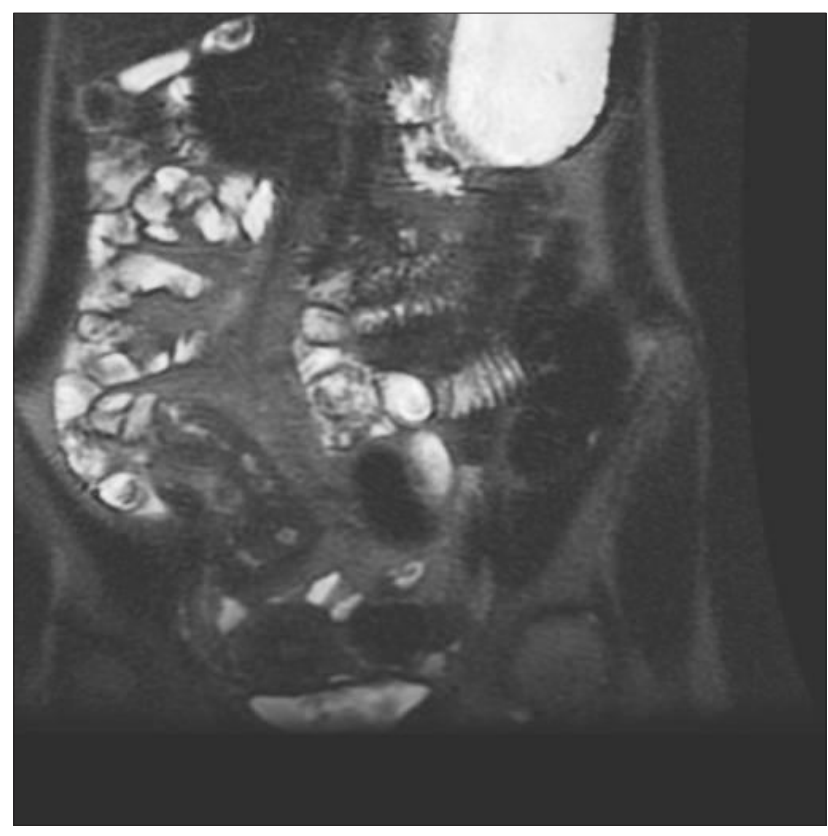

Fig. 1. MREg, 17 year woman, chronic Crohn's disease, coronal plane, T2SSFSE + biphasic PEG oral solution - homogeneous hyperintensive intraluminal filling of the small bowel, fibrotic inflammatory changes at the terminal ileum, extraluminal changes are not seen.

MRI diagnostics, e.g. AMI-121 (ferumoxsil Lumirem and Gastromark, Malinckrodt) (Fig. 3,4), OMP (Abdoscan, Nycomed,Amershem), perfluorooctylbromide (Imagent GI), perfluorocarbon ${ }^{7-9}$. Ultrasmall Superparamagnetic Iron Oxides (USPIO) for liver/spleen MR imaging are experimentally used, e.g. AMI-25 (Endorem and Feridex IV), SHU 555A (Resovist). Smaller iron oxide particles are selected for lymph node imaging (AMI-227 - Sinerem and Combidex), bone marrow imaging (AMI-227), perfusion imaging (NC100150 - Clariscan) and MR angiography $(\mathrm{NC} 100150)\left(\right.$ ref. $\left.^{10}\right)$.

SPIO nanoparticles are strong proton enhancers resulting in shortening both $\mathrm{T} 1$ and $\mathrm{T} 2$ relaxation times and thus by dephasing spins (susceptibility effect) superparamagnetic particles cause decrease in the signal intensity on T2 weighted images ${ }^{9}$. Negative contrast agents decrease noise and motion artifacts related to the bowel peristalsis and improve the detection of intramural edema and extramural complications (necrosis, fat edema, mesenterial edema, abscess formation or fistulas) (ref. ${ }^{11,12}$ ). SPIO negative contrast agents are appropriate for MRCP as they decrease the non-homogenous signal of the bowel lumen and the desired organ such as biliary duct or pancreas can be better visualized. Currently commercial oral bowel negative contrast agents are still not used routinely due to the high investigational costs. Kluchova, et al. described and provided a new negative contrast agent composed of superparamagnetic nearly monodispersed maghemite nanoparticles prepared by thermal induced solid-state reaction from iron (II) acetate in air that are incorporated in a layered aluminosilicate mineral (bentonite matrix). Bentonite is a natural, low-

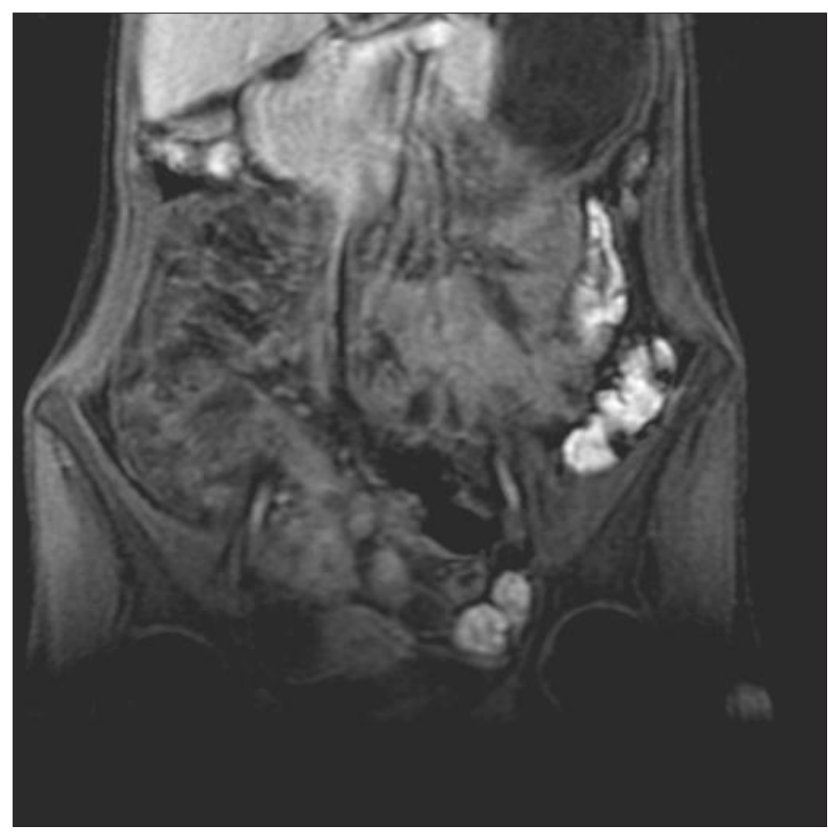

Fig. 2. MREg, 17 year woman, chronic Crohn's disease, coronal plane, 3D T1fSPGR fat suppresion+ biphasic PEG oral solution - hypointensive intraluminal filling of the small bowel, pathologic fibrotic changes at the terminal ileum without extraluminal fluid.

cost, readily available and biocompatible aluminosilicate mineral endowed with unique swelling, sorpsion, ion exchange and intercalation properties. The clay is known to be essentially nontoxic and established by the Food and Drug Administration as "Generally Recognized As Safe" (GRAS). The detailed process of synthesis, physical and chemical characterization of this new oral negative SPIO contrast agent is reported by Kluchova et al. ${ }^{8}$.

The aim of this study was to test the experimental SPIO oral solution - maghemite in MRI of healthy volunteers,patients with chronic inflammatory small bowel diseases, and MRCP.

\section{PATIENTS AND METHODS}

\section{Patients}

The study was conducted in cooperation of the Department of Radiology, F.D.Roosevelt Faculty Hospital Banska Bystrica (Slovak Republic) with the Regional Center of Advanced Material, Palacky University, Olomouc (Czech Republic) between March 2008 and October 2009. All participating volunteers and patients signed informed consent approved by ethics committee.

The experimental oral negative contrast solution was tested in 3 cohorts: 17 healthy volunteers (group A) underwent MREg, 24 patients with small bowel disease underwent MRCP (group B) and 22 patients with small bowel disease (group C) underwent MREg. Volunteers and patients fasted 6-8h before examination. Characteristics of the study samples are summarized in (Table 1). There was no significant differences in gender between groups 
Table 1. Characteristics of studied population $(n=63)$.

\begin{tabular}{lc}
\hline Characteristics & Number \\
\hline Gender male/female & $21 / 42$ \\
Age, median (range), years & $53.5(19-88)$ \\
Healthy volunteers (group A) & 17 \\
Patients with MRCP (group B): & 24 \\
- liver cirrhosis before liver transplantation & 5 \\
- chronic pancreatitis & 3 \\
- acute pancreatitis & 2 \\
- cholangitis & 2 \\
- gallstones & 3 \\
- benign stenosis of biliary tract & 1 \\
- malignant stenosis of biliary tract & 1 \\
- primary sclerosing cirrhosis (PSC)+ Crohn's disease & 3 \\
- non-diagnosed renal cell carcinoma with metastases & 1 \\
- dyspepsia after cholecystectomy & 2 \\
- chronic hepatitis & 1 \\
Patients with MREg (group C): & 22 \\
- non-specific enteritis & 1 \\
- Crohn's disease & 9 \\
- celiac disease + chronic small bowel inflammation & 2 \\
- celiac disease + Crohn's disease & 1 \\
- bowel edema in liver cirrhosis before liver transplantation & 3 \\
- Crohn's disease + liver cirrhosis + Turner syndrome & 1 \\
- Crohn's disease +PSC & 1 \\
- primary biliary cirrhosis (PBC) & 1 \\
- diarrhoe in leukemia & 1 \\
- non-specific diarrhoe & 1 \\
- chronic pancreatitis & 1 \\
\hline
\end{tabular}

Footnotes:MRCP-magnetic resonance cholecystopancreaticography, MREg- magnetic resonance enterography

$(P=0.974)$. Statistical analysis proved a significantly higher median age in group B (54.5 years) compared to group A (39 years, $P=0.001$ ) and group $\mathrm{C}$, respectively (36 years, $P=0.002)$.

All patients and volunteers completed questionnaires evaluating the solution characteristics (amount, taste, characteristic of taste, appearance). A four-step scoring system was used to evaluate the amount of experimental solution (1.sufficient, 2.excessive, 3.insufficient, 4.unknown) evaluated by patients and volunteers, taste (1.perfect, 2.good, 3.bad, 4.another-specify), characteristic of taste (1.fruity, 2.metallic, 3.synthetic, 4.another-specify) and appearance (1.perfect, 2.good-looking, 3.bad-looking, 4.another-specify).

Each of monitored side-effects (nausea, eructation, flatulence, diarrhoea) during and $2 \mathrm{~h}$ after drinking was evaluated by either point 0 (no side-effects) or point 1 (side-effect present - without severity assessment).

\section{Oral contrast agent solution}

The tested experimental solution (1000 ml) was composed of: maghemite nanoparticles $800 \mathrm{mg}$, bentonite matrix 4 g, fruit juice $500 \mathrm{ml}$ - apple/carrot (HAMI, Nutricia,
$\mathrm{CZ}$ ), water $500 \mathrm{ml}$, polyethylene glycol 4000 (Macrogol) $76.8 \mathrm{~g}+2.82 \mathrm{~g}$ sodium sulphate $\left(\mathrm{Na}_{2} \mathrm{SO}_{4}\right)+0.84 \mathrm{~g}$ sodium bicarbonate $\left(\mathrm{NaHCO}_{3}\right)+0.72 \mathrm{~g}$ sodium chloride $(\mathrm{NaCl})+0.36 \mathrm{~g}$ potassium chloride $(\mathrm{KCl})$. Fruit juice acts against the nanoparticle sedimentation in the bowel and is useful for homogenic lay out of solution in the bowel. Additionally, the taste of the experimental solution is further improved by the fruit juice.

\section{Study assessment}

To define the best signal intensity (SI) of the intraluminal lay out the maghemite concentrations 200, 400, 600,800 and $1000 \mathrm{mg} / 4 \mathrm{~g}$ bentonite of the experimental solution were tested in healthy volunteers (group A). The final concentration of the experimental solution was compared to the commercially available solution ferumoxsil (Lumirem, Guerbet, France) in group A (Fig. 3,4,5,6). Four specialists in gastrointenstinal MRI evaluated all MR results in studied groups.

Groups A and C drank $1000 \mathrm{ml}$ of experimental solution with maghemite fractionally within $50 \mathrm{~min}$ followed by MREg without intravenous contrast agent and spasmolytics. Group B drank $500 \mathrm{ml}$ of experimental solution 


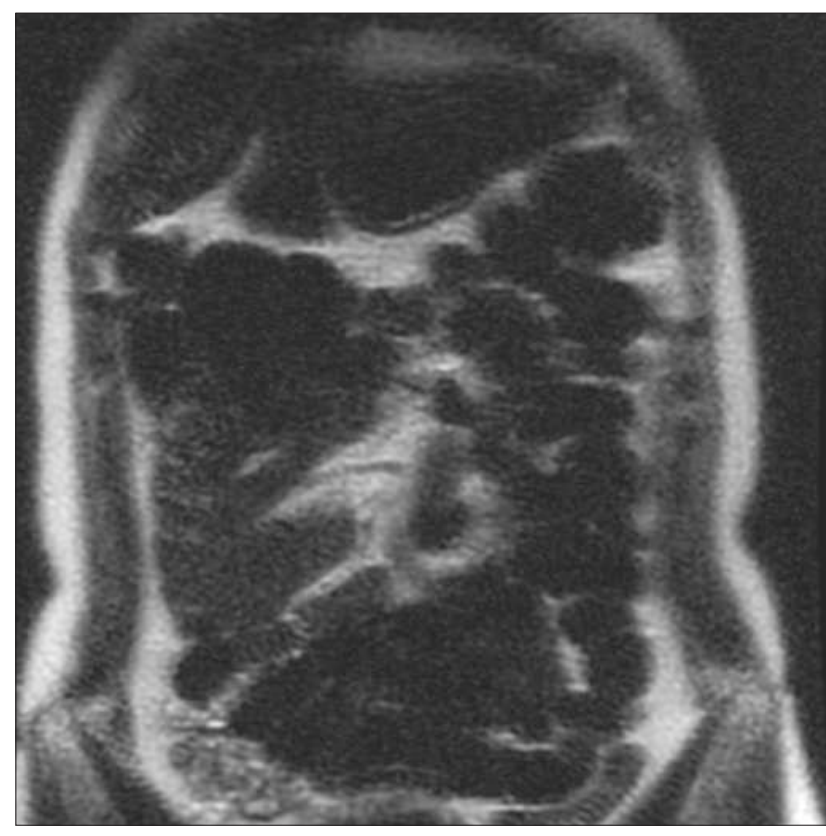

Fig. 3. MREg,volunteer, coronal plane, T2SSFSE + Lumirem oral solution - homogeneous negative intraluminal filling of the small bowel.

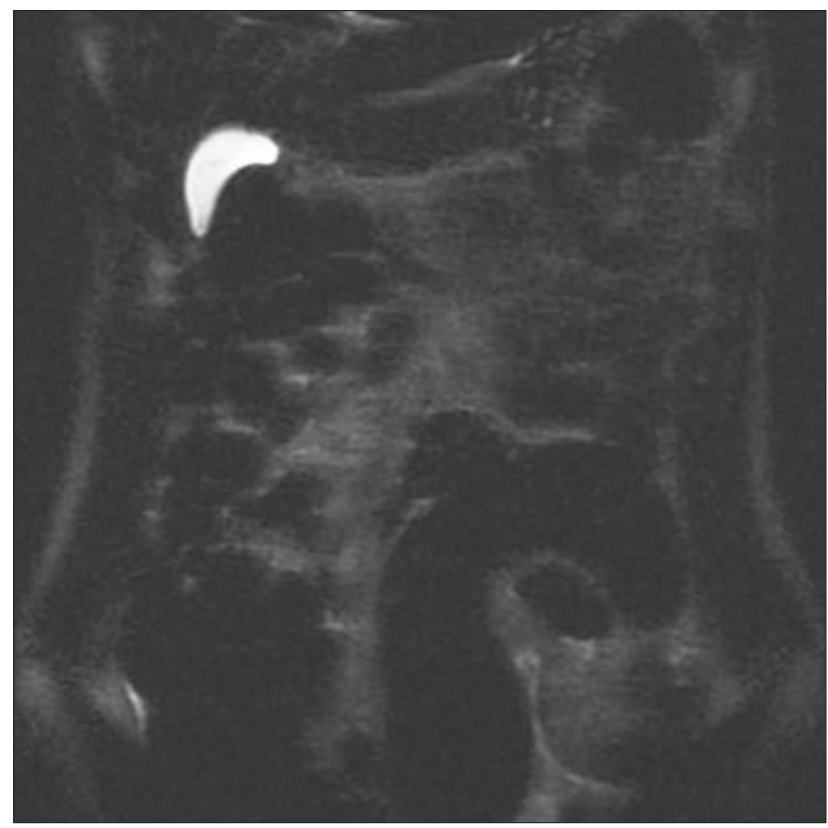

Fig. 5. MREg, volunteer, coronal plane, T2SSFSE + experimental SPIO maghemit oral solution - homogeneous negative intraluminal filling of the small bowel.

fractionally within 30 min followed by MRCP without intravenous contrast agent and spasmolytics.

\section{Imaging protocol}

All examinations were realised on the MR unit $1.5 \mathrm{~T}$ system (Signa Horizon Lx, GE, Milwaukee,Wisconsin, USA), with body phased array, in prone position. Protocol of MREg with experimental maghemite oral solution (group A,C): localiser, breath-hold axial plane 2D T2 single-shot fast spine-echo (SSFSE) TE 80, TR

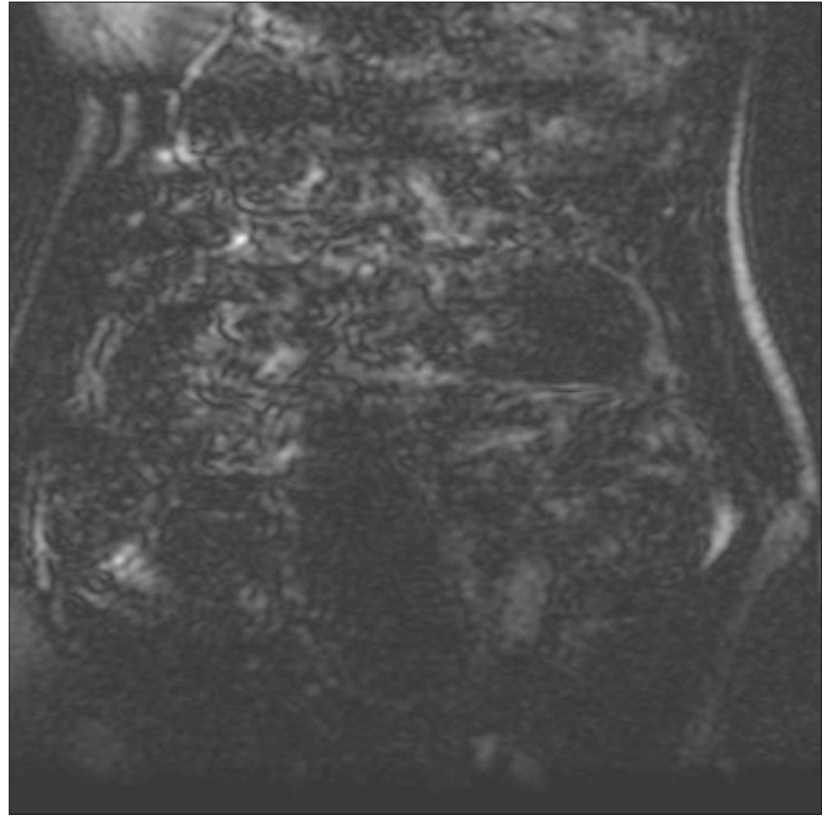

Fig. 4. MREg, volunteer, coronal plane, 3DT1fSPGR fat supression + Lumirem oral solution- inhomogeneous hypointensive intraluminal filling of the small bowel.

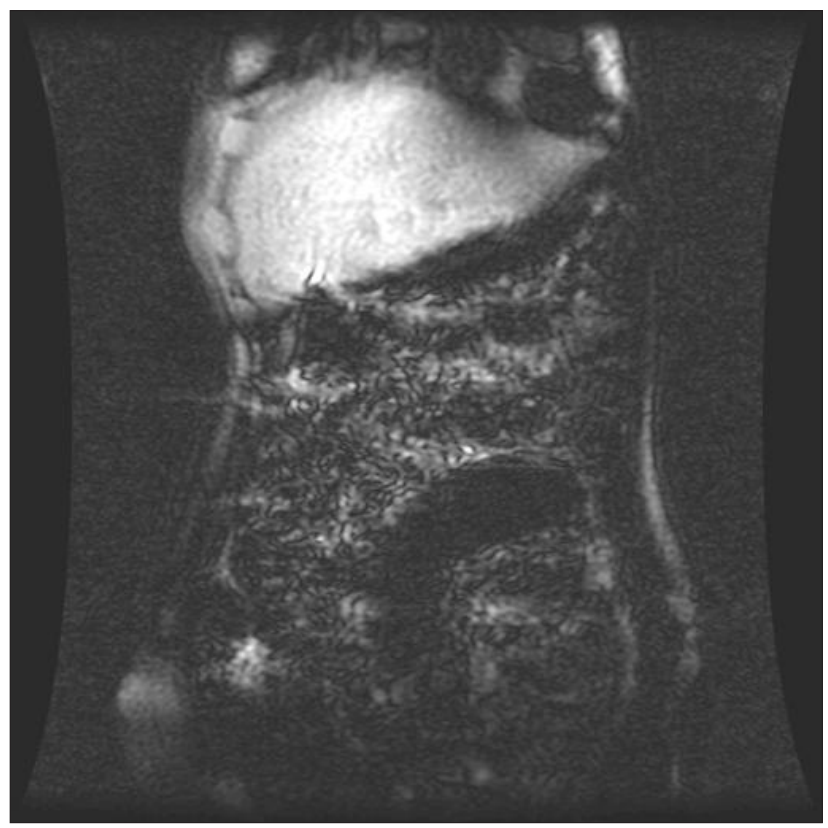

Fig. 6. MREg, volunteer, coronal plane, 3D T1 fSPGR fat supression + experimental SPIO maghemit oral solution - inhomogeneous hypointensive intraluminal filling of the small bowel.

minimum=1852, FOV $38 \times 38 \mathrm{~cm}$, bandwith 31.25 , slice thickness $5 \mathrm{~mm}$, sp 1.0 matrix 256 x 256, breath-hold coronal plane 2D T2 SSFSE TE 80 , TR minimum=2027, bandwith 31.25 FOV 48 x $48 \mathrm{~cm}$, matrix 384 x 320, sp 1.0, slice thickness $5 \mathrm{~mm}$. Coronal plane T1 3D fast spoiled gradient echo (fSPGR) fat suppressed, coronal plane 3D Fast Imaging Employing Steady State Aquistition (FIESTA), without intravenous contrast agent and spasmolytics. Group B underwent MRCP protocol after 30 min of drinking experimental oral maghemite solution: 
Table 2. Side effects during and 2 hours after drinking in patients and volunteers $(n=63)$.

\begin{tabular}{|c|c|c|c|c|c|c|c|c|}
\hline \multirow{3}{*}{ Side effects } & \multicolumn{4}{|c|}{ Side effects during drinking } & \multicolumn{4}{|c|}{ Side effects 2 hours after drinking } \\
\hline & \multicolumn{4}{|c|}{$\begin{array}{l}\text { Number of patients/volunteers } \\
\qquad(\%)\end{array}$} & \multicolumn{4}{|c|}{$\begin{array}{l}\text { Number of patients/volunteers } \\
\qquad(\%)\end{array}$} \\
\hline & Group A & Group B & Group C & Total & Group A & Group B & Group C & Total \\
\hline $\begin{array}{l}\text { No side } \\
\text { effects }\end{array}$ & $\begin{array}{c}6 \\
(35.3)\end{array}$ & $\begin{array}{c}18 \\
(75.0)\end{array}$ & $\begin{array}{c}9 \\
(40.9)\end{array}$ & $33(52.4)$ & $\begin{array}{c}3 \\
(17.6)\end{array}$ & $\begin{array}{c}8 \\
(100)\end{array}$ & $\begin{array}{c}8 \\
(36.4)\end{array}$ & $\begin{array}{c}19 \\
(40.4)\end{array}$ \\
\hline Nausea & $\begin{array}{c}5 \\
(29.4)\end{array}$ & $\begin{array}{c}2 \\
(8.3)\end{array}$ & $\begin{array}{c}2 \\
(9.1)\end{array}$ & $\begin{array}{c}9 \\
(14.3)\end{array}$ & $\begin{array}{c}3 \\
(17.6)\end{array}$ & $\begin{array}{c}0 \\
(0)\end{array}$ & $\begin{array}{c}0 \\
(0)\end{array}$ & $\begin{array}{c}3 \\
(6.4)\end{array}$ \\
\hline Eructation & $\begin{array}{c}5 \\
(29.4)\end{array}$ & $\begin{array}{c}1 \\
(4.2)\end{array}$ & $\begin{array}{c}0 \\
(0)\end{array}$ & $\begin{array}{c}6 \\
(9.5)\end{array}$ & $\begin{array}{c}2 \\
(11.8)\end{array}$ & $\begin{array}{c}0 \\
(0)\end{array}$ & $\begin{array}{c}0 \\
(0)\end{array}$ & $\begin{array}{c}2 \\
(4.3)\end{array}$ \\
\hline Flatulence & $\begin{array}{c}1 \\
(5.9)\end{array}$ & $\begin{array}{c}2 \\
(8.3)\end{array}$ & $\begin{array}{c}5 \\
(22.7)\end{array}$ & $\begin{array}{c}8 \\
12.7)\end{array}$ & $\begin{array}{c}1 \\
(5.9)\end{array}$ & $\begin{array}{c}0 \\
(0)\end{array}$ & $\begin{array}{c}2 \\
(9.1)\end{array}$ & $\begin{array}{c}3 \\
(6.4)\end{array}$ \\
\hline Diarrhoea & $\begin{array}{c}0 \\
(0)\end{array}$ & $\begin{array}{c}1 \\
(4.2)\end{array}$ & $\begin{array}{c}6 \\
(27.3)\end{array}$ & $\begin{array}{c}7 \\
(11.1)\end{array}$ & $\begin{array}{c}8 \\
(47.1)\end{array}$ & $\begin{array}{c}0 \\
(0)\end{array}$ & $\begin{array}{c}12 \\
(54.5)\end{array}$ & $\begin{array}{c}20 \\
(42.6)\end{array}$ \\
\hline
\end{tabular}

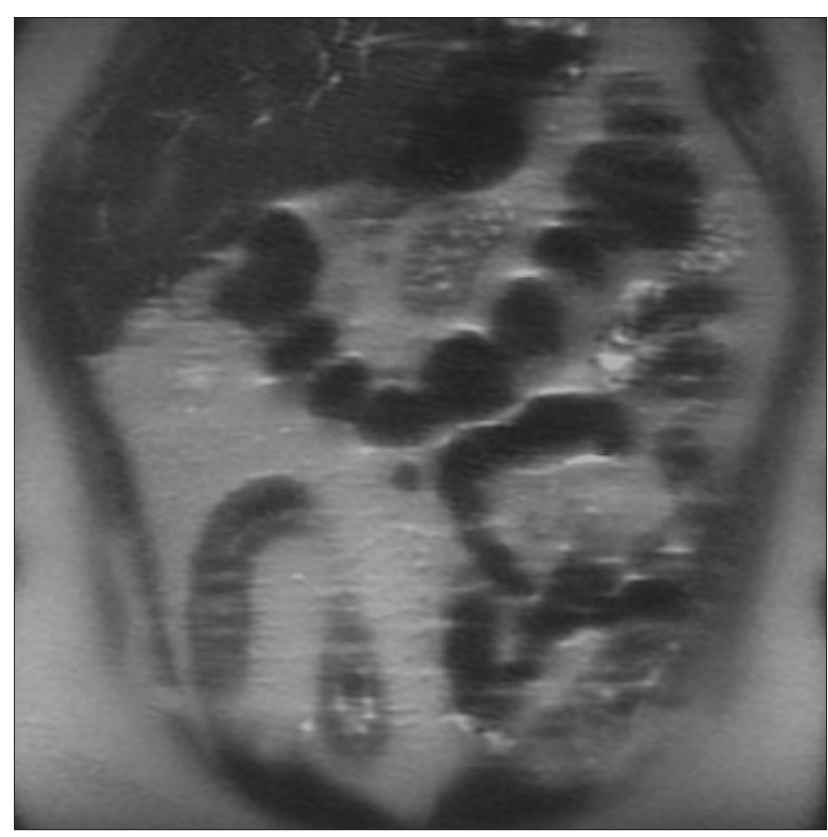

Fig. 7. MREg, 61 year woman, chronic Crohn's disease, coronal plane, T2SSFSE + experimental SPIO maghemit oral solution - homogeneous negative intraluminal filling of intact loops of the small bowel, fibrotic changes in affected loop of the terminal ileum without extraluminal changes.

localiser, axial/coronal planes 2D T2 SSFSE TE 500, TR6000, bandwith 31.25, matrix 384 x 320, FOV 36, coronal plane 3D FIESTA, MRCP- slice thickness $20 \mathrm{~mm}$, spacing 0 , number of slices 14 and more.

All image analyses were performed on the workstation ADW 4.5 (GE, Milwaukee,Wisconsin, USA).The matrix size and resolution of the monitors were $1500 \times 2000$ (3 megapixels).

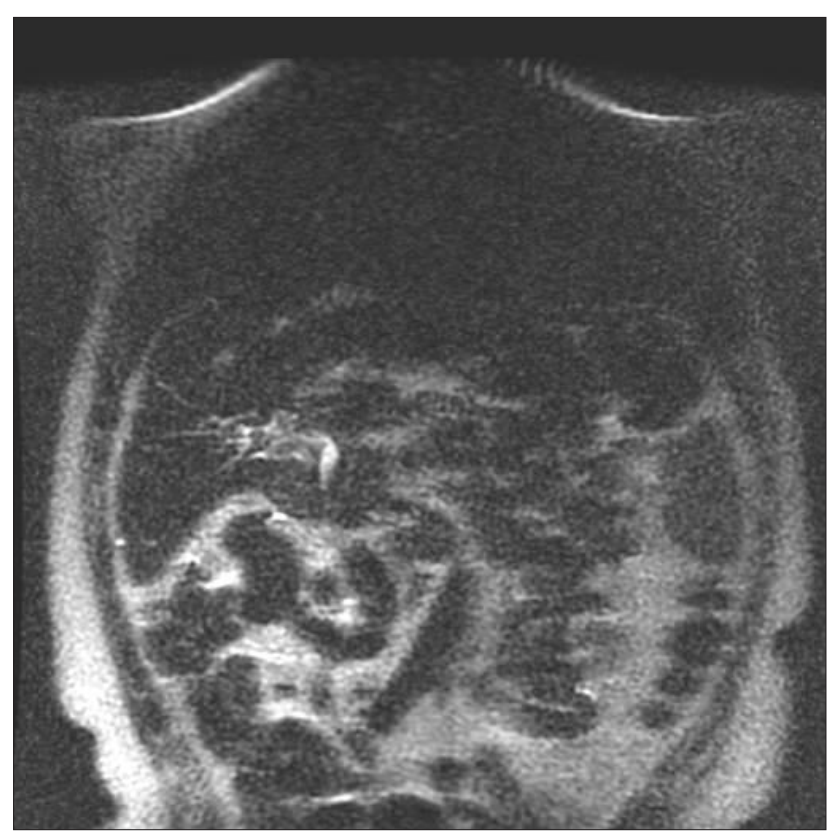

Fig. 8. MRCP,coronal plane,T2SSFSE + experimental negative SPIO oral solution, liver cirrhosis, free intraabdominal fluid, edema of the bowel wall due to portal hypertension, better delineation of the small bowel wall in the fluid.

\section{Statistics}

Pearson's chi-square test and Fisher's exact test were used for the evaluation of categorical variables. Quantitative parameters were analysed by KruskalWallis test and nonparametric Mann-Whitney test with Bonferroni correction was used for analysis of significant results. All tests were made at the level of significance $P \leq 0.05$. Data were analyzed by SPSS v. 15 software (SPSS Inc., Chicago, USA). 


\section{RESULTS}

Based on visual assessment of four MRI specialists the concentration of $800 \mathrm{mg}$ maghemite/4 $\mathrm{g}$ bentonite in $1000 \mathrm{ml}$ of the experimental solution was optimal for proper intramurally lay-out in all 3 groups. The concentration of $800 \mathrm{mg}$ maghemite/4 $\mathrm{g}$ bentonite was comparable to ferumoxsil (Lumirem, Guerbet) for MREg in group A according to visual assessment (Fig. 5,6).

The amount of experimental solution $1000 \mathrm{ml}$ vs 500 $\mathrm{ml}$ evaluated by patients and healthy volunteers according to scoring system was not statistically significant between groups $(P=0.085)$. The taste of the solution did not differ significantly in groups $\mathrm{A}, \mathrm{B}$ and $\mathrm{C}(P=0.097)$, however, $20 \%$ of the women across the groups described a bad taste of the solution. There was no significant difference in characteristic of the taste among groups $\mathrm{A}, \mathrm{B}$ and $\mathrm{C}$ $(P=0.298)$. The tested solution appearance was not found to be significant in all groups $(P=0.319)$. The relationship between the taste, characteristic of the taste and appearance was statistically significant across the groups: fruity taste was associated with good taste and good-looking appearance $(P=0.05)$; metallic taste was associated with bad taste and bad-looking appearance $(P=0.05)$.

Side-effects during and $2 \mathrm{~h}$ after drinking in all groups are summarized in (Table 2). The most common sideeffects during drinking of the experimental solution were nausea $(29.4 \%)$ and eructation $(29.4 \%)$ in group A and diarrhoea $(27.3 \%)$ in group C.

Side-effects $2 \mathrm{~h}$ after drinking the experimental solution occured in group A (nausea 17.6\%) and in group $\mathrm{C}$ (diarrhoea 54.6\%). Once the side effects occured during drinking, they persisted for up to $2 \mathrm{~h}$ after drinking $(P=0.05)$. None of the patients in group B suffered from problems $2 \mathrm{~h}$ after drinking the experimental solution. Based on the evaluation of all side effects during and $2 \mathrm{~h}$ after drinking the best tolerated experimental solution was in group B when compared to group A $(P=0.0003)$ and to group $\mathrm{C}(P=0.002)$, respectively. Side-effects of the solution in groups $\mathrm{A}$ and $\mathrm{C}$ did not differ significantly. The Spearman correlation analysis showed a significant relationship between overall evaluation of experimental solution and age: the older subjects assessed the solution more favorably than the younger one $(r / y=-0.482)$. The best tolerance of the experimental solution was found in the group B with significantly higher median age of patients age than groups $\mathrm{A}$ and $\mathrm{C}$.

\section{DISCUSSION}

Superparamagnetic iron oxide MR imaging contrast agents have been the subject of extensive research over the past decade ${ }^{10}$. Iron oxide particles for bowel contrast are coated with insoluble material, and all iron oxide particles for intravenous injection are biodegradable. Superparamagnetic agents open up an important field for research in MR imaging. Rieber et al. used positive and negative oral contrast agents in MREc for better de- lineation of interloop abscesses in Crohn's disease. T2weighted sequences were necessary using negative oral contrast media, because loop abscesses may be masked. Magnetic resonance imaging should be performed in all patients with a suspicion of extraintestinal complications, because the complications are more reliably detected by MRI (Fig. 7). Negative oral contrast media show advantages with the use of intravenous contrast but can mask loop abscesses using only T1-weighted imaging ${ }^{13}$. A new superparamagnetic iron oxide contrast agent ferristene is a safe and effective endoluminal contrast medium used for magnetic resonance (MR) enteroclysis ${ }^{14}$. This study evaluated the applicability of experimental oral contrast solution with maghemite for MRCP and MREg examinations in patients with small bowel diseases.

Based on visual assessment and the consensus of four MRI specialists, the concentration of $800 \mathrm{mg}$ maghemite/4 g bentonite in $1000 \mathrm{ml}$ of experimental solution was optimal for the proper intramurally lay-out required for MREg and MRCP (Fig. 8) in all 3 groups. In MREg this concentration of experimental solution was comparable with the commercial solution ferumoxsil (Lumirem,Guerbet). Both solutions are negative contrast agents based on superparamagnetic iron oxide complexes. T2-weighted MR with superparamagnetic oral contrast agent ferumoxsil is used in Crohn's disease and helps to assess mural and transmural inflammation with the same accuracy as gadolinium-enhanced T1-weighted MR (Fig. 7). The combination of gadolinium-enhanced T1and T2-weighted sequences is useful in the assessment of Crohn's disease ${ }^{2}$. In our experience the solution with maghemite is suitable for MREg examination for all tested indications: non-specific enteritis, Crohn's disease, celiac disease, chronic small bowel inflammation, changes of small bowel in liver diseases, acute and chronic pancreatitis.

The experimental solution of $500 \mathrm{ml}$ was used for MRCP examination to suppress the signal intensity in the proximal loops of the small bowel and for better depiction of the biliary tree especially in liver cirrhosis and free intra-abdominal fluid (Fig. 8). The MRI specialists generally recommend $1000 \mathrm{ml}$ of the experimental solution to depicting of the whole small bowel. There were no significant differences in the evaluation of experimental solution quantity( $1000 \mathrm{ml}$ vs $500 \mathrm{ml}$ ) based on questionnaires from patients and healthy volunteers.

Fruit juice was added to the experimental oral contrast solution to improve its taste. The difference in taste was not peceived over groups, but the taste perception was gender specific: $20 \%$ of women across the groups described a bad solution taste. The characteristic of taste and appearance of experimental solution did not differ significantly among groups. We analysed the relationship among the taste, characteristic of the taste and appearance and we found a significant correlation of fruity taste with good taste and good-looking appearance. In contrast, metallic taste was associated with bad taste and bad-looking appearance. Most of the side-effects (nausea, eructation and diarrhoea) occured during drinking of the 
experimental solution in groups A and C. Once the sideeffects occured during drinking, they persisted up to $2 \mathrm{~h}$ after drinking in both groups A and C. Group B tolerated experimental solution with minimum of side-effects. Better evalution of the solution in the group B was influenced by the older age of these patients than groups A and C. Interestingly, volunteers and patients in groups A and $\mathrm{B}$ aged over 50 years did not complain of problems during and after drinking. This could be partly explained by the fact that in general the older population covers up information about their health problems. Side effects of the experimental solution did not differ between the healthy volunteers (group A) and patients with small bowel diseases (groups B,C). On T1-weighted images simultaneous use of negative oral contrast agents and intravenous injection of gadolinium-chelate produces the best conditions for double-contrast techniques. The combination of negative oral contrast agents and intravenous injections of gadolinium chelate increases significantly the contrast between the wall and pathological findings in the wall (inflammatory changes and tumors) $\left(\right.$ ref. $^{7}$ ).

\section{CONCLUSION}

The experimental maghemite solution including quantity, taste and appearance was well- tolerated in all studied groups. Our study supports the use of this solution in MRI practice. More studies are needed to confirm our results in adult and pediatric patients with small bowel diseases.

\section{ACKNOWLEDGEMENT}

Radiologists - MR specialists: Katarina Novotna, Radoslav Bielik, Richard Mihalik, Department of Radiology, F.D.Roosevelt Faculty Hospital Banska Bystrica, Slovak Republic. Pavel Novak, MR center MEDIHOPE s.r.o., Prostejov, Czech Republic.

This research was supported by IGA UP Olomouc grant number LF 2011007 and grant number CEP 1 MO5512.

The study was approved by the local ethics committee of Faculty Hospital F.D.Roosevelt in Banska Bystrica (Slovak Republic). All patients were aged over 18 years and signed informed consent with the study prior to data collection.

The authors gratefully acknowledge to the project OPVK 2.3 (CZ.1.07/2.3.00/20.0017) - Research team development of the Regional Centre of Advanced Technologies and Materials (RCPTM-TEAM)

\section{CONFLICT OF INTEREST STATEMENT}

Author's conflict of interest disclosure: The authors stated that there are no conflicts of interest regarding the publication of this article.

\section{REFERENCES}

1. Maglinte DD, Siegelman ES, Kelvin FM. MR enteroclysis:the future of small-bowel imaging. Radiology 2000;215(3):639-41.

2. Maccioni F, Bruni A, Viscido A, Colaiacomo MC, Cocco A, Montesani C,Caprilli R, Marini M. MR imaging in patiens with Crohn's disease: value of T2 - versus T1 - weighted gadolinium - enhanced MR sequences with use of an oral superparamagnetic contrast agent. Radiology 2006;238(2):517-30.

3. Prassopoulos P, Papanikolaou N, Grammatikakis J, Rousomoustakaki M,Mari T, Gourtsoyiannis N. MR enteroclysis imaging of Crohn's disease. Radiographics 2001;21:S61-72.

4. Bruining DH, Loftus EV. Crohn's disease clinical issues and treatment: what the radiologist needs to know and what the gastroenterologist wants to know. Abdom Imaging 2009;34:297-302.

5. Gourtsoyiannis N, Papanikolaou N, Grammatikakis J, Prassopoulos P. MR enteroclysis: technical considerations and clinical applications. Eur Radiol 2002;12(11):2651-8.

6. Siddiki H, Fidler J. MR imaging of the small bowel in Crohn's disease. Eur J Radiol 2009;69:409-17.

7. Giovagnoni A, Fabbri A, Maccioni F. Oral contrast agents in MRI of the gastrointestinal tract. Abdom Imaging 2002;27(4):367-75.

8. Kluchova K, Zboril R, Tucek J, Pecova M, Zajoncova L, Safarik I, Mashlan M, Markova I, Jancik D, Sebela M, Bartonkova H, Bellesi V, Novak P, Petridis D. Superparamagnetic maghemit nanoparticles from solid - state synthesis - their functionalization towards peroral $\mathrm{MRI}$ contrast agent and magnetic carrier for trypsin immobilization. Biomaterials 2009;30:2855-63.

9. Patak MA, vonWeyman C, Froehlich JM. Small bowel MR imaging:1.5T versus 3T. Magn Reson Imaging Clin N Am 2007;15(3):383-93.

10. Wang $Y X$, Hussain SM, Krestin GP. Superparamagnetic iron oxide contrast agents: physicochemical characteristics and applications in MR imaging. Eur Radiol 2001;11(11):2319-31.

11. Narin B, Aja W, Göhde S, Langhorst J,Akgoz H, Gerken G, Ruhm SG, Lauenstein TC. Combined small and large bowel MR imaging in patients with Crohn's disease: a feasibility study. Eur Radiol 2004; 14:1535-42.

12. Debatin JF, Patak MA. MRI of the small and large bowel. Eur J Radiol 1999;9:1523-34.

13. Rieber A, Aschoff A, Nussle K, Wruk D, Tomczak R, Reinshagen M, Adler G, Brambs HJ. MRI in the diagnosis of small bowel disease: use of positive and negative oral contrast media in combination with enteroclysis. Eur Radiol 2000;10(9):1377-82.

14. Boraschi P, Braccini G, Gigoni R, Cartei F, Perri G. MR enteroclysis using iron oxide particles (ferristene) as an endoluminal contrast agent: an open phase III trial. Magn Reson Imaging 2004;22(8):1085-95. 